\title{
A Review of Supervised Variational Quantum Classifiers
}

\author{
Amogh Agastya ${ }^{1}$, Dhruv Saldhana ${ }^{2}$, Pratyanush $\mathrm{G}^{3}$, Prof. Madhavi R P ${ }^{4}$ \\ 1,2,3 U.G Students, ${ }^{4}$ Associate Professor, Computer Science and Engineering Department, BMSCE, \\ Bengaluru, Karnataka, India.
}

\begin{abstract}
Innovation in machine learning is far from complete. In fact, things are just about to take a 'quantum leap', when the world of quantum physics and machine learning are about to merge, aiming to solve advanced problems through intelligent computing. Quantum-classical hybrid techniques termed "variational algorithms" are well-suited to noisy quantum computing devices and are swiftly gaining popularity in the quantum machine learning community. Quantum machines tap directly into an unimaginably vast fabric of reality - the unusual and counterintuitive realm of quantum field theory, to accelerate computation. Quantum computers will predictively play an important role in machine learning, including the essential element of accessing more computationally complex feature spaces.
\end{abstract}

Keywords- Quantum Machine Learning, Quantum Classification, Hybrid Quantum Algorithm

\section{INTRODUCTION}

Quantum computing is the practical application of quantum phenomena such as superposition and entanglement to do computation that can be theoretically or physically applied [1]. This can be verified both theoretically and experimentally. The present time is now known as the Noisy Intermediate-Scale Quantum era, commonly referred to as the NISQ era [13]. Specifically, this refers to quantum devices of approximately less than 100 qubits whose quantum logic gates of which still bear some error with them. Machine learning is a way to determine and evaluate patterns (instead of using traditionally-coded algorithms) and can be applied to a variety of different applications, however, its implementation in Artificial Intelligence (AI) is the one that's got the whole world abuzz. We are interested in this intersecting field where quantum computers apply machine learning algorithms and conventional machine learning methods are employed to assess the quantum machines. This area of research is developing at such blazing speeds that it has spawned an entire new field called Quantum Machine Learning (QML).

Classical computer are based on an architecture that works on binary state's that is 1 's and 0 's, each signifying a given state. In a Quantum environment there exists multiple states, that is 1 's, 0 's and both which signify respective states the machine can be in at any given state at any given time and thus is the definition of superposition. A Quantum circuit is at the heart of the Quantum hardware, it handles execution in a sequential form using Quantum Gates placed in a continual format. The Quantum Mechanical Phenomenon's are used to extrapolate and manipulate the outputs and hence we will use Quantum
Entanglement and Quantum Tunneling to get the state. This enables Quantum Hardware Devices to explore multiple states all at once and at a very high speed. Here Quantum Machine learning involves classical algorithms which when executed help to solve problems, these process uses Quantum Hardware which requires the classical algorithm to be mapped into a Quantum understandable form. This helps make the training faster and testing more accurate in labelling the Iris Flower data set. The results of these processes involves measuring Qubits at the end of processing which will give us our classification of the Iris Flower.

Many Quantum algorithms have been executed on NearTerm Quantum Devices, these are basically Application Specific IC devices or Field Programmable hardware. This is due to the fact that full-fledged Quantum Hardware is a thing of the future until then we cannot talk about the Quantum Advantages. Hence the best practice or the logical way to go about executing Quantum Algorithms is to encode the classical data into amplitudes of Quantum states, this with the help of Quantum Circuits will allow for execution [7, 8]. The Quantum Algorithms having ' $n$ ' Qubits can be encoded into $2^{\text {n }}$ respective amplitudes, this means that the execution will be in poly-arithmetic time $[9,12]$.

The present progress of Quantum Computing has given rise to Noisy Intermediate- Scale [13], this is basically a Quantum Computer with less than 100 Qubits and will contain some noise or error in the result. These devices having less than 100 Qubits and an error coefficient will not be suitable for large scale execution of everyday problems until a fully functional Quantum Environment can be created hence algorithms must be structured in such a way to be unaffected by errors. Variational Quantum algorithms are conceptualized on the principle of dual nature ${ }^{[14]}$, that is a Quantum Circuit keeps updating the parameters based on some rule depending on the output and executes the algorithm with the parameterized requirements, these circuits work in an iterative fashion such that when an output is produced the circuit tries to optimize itself by manipulating its parameters in accordance to some rule.

The next section elaborates on Variation Quantum Classifier (VQC). 


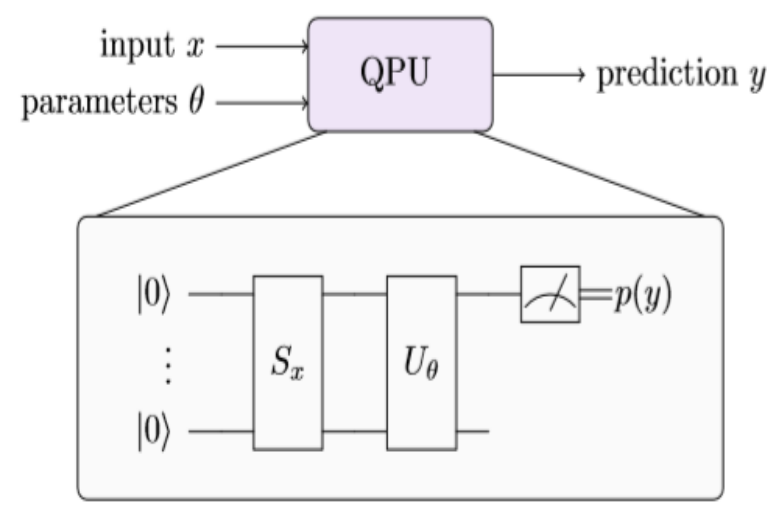

FIG. 1. Idea of the circuit-centric quantum classifier. Inference with the model $f(x, \theta)=y$ is executed by a quantum device (the quantum processing unit or QPU) which consists of a state preparation circuit $S_{x}$ encoding the input $x$ into the amplitudes of a quantum system, a model circuit $U_{\theta}$, and a single qubit measurement. The measurement retrieves the probability of the model predicting 0 or 1 , from which in turn the binary prediction can be inferred. The classification circuit parameters $\theta$ are learnable and can be trained by a variational scheme.

\section{VARIATIONAL QUANTUM CLASSIFIER (VQC)}

Noisy Intermediate-Scale Quantum or NISQ a Variational Quantum Classifier (VQC) is a hybrid made up of Quantum and Classical algorithm where "Layers" or "blocks" the basic Quantum building block are repeatedly used to form Variational Quantum Classifier Circuits which will be the executor or processor in this demonstration, it must be noted that it should resilient to errors. The architecture is inspired by Schuld et al. (2018) and Farhi and Neven (2018). Elies M. Gil Fuster, Variational Quantum Classifier.

In this paper we will demonstrate that a Variational Quantum Classifier behavior will be determined by Quantum Circuits and output dependent functions based on free parameters executed iteratively in comparison to classical execution which takes place iteratively to minimize this functions outcome which will also make it the fault tolerant. The classical execution involves something call gradient descent, this basically means it tries to find the local minima of a function. The Quantum execution for supervised learning works differently in that it make use of Variational Algorithms which are implemented using differential programing, State Preparation which encodes classical data sets into amplitude and rotations of Quantum bits for Quantum hardware to understand after this the Quantum bits are executed using parameterized Unitary Operations, all parameters may by modified depending on given rules, the output which is the classification of the Iris Dataset is to be finally measured when the job is done.

\section{A. State Preparation}

In State Preparation an initial input ' $x$ ' has to be encoded using amplitude encoding of Quantum bits, this is done by applying the static state circuit to map the classical dataset into it respective Quantum Qubits such that it is understood by the Quantum Circuit and is then executed in the initial ground state. The output Qubits are measured to get the resultant prediction of classification of the Iris flower. This is better understood by mapping ' $n$ ' qubit Quantum Circuit Architecture where an input ' $x$ ' $\in R$ to the $2^{\mathrm{n}}$ amplitude vector $\phi(\mathrm{x})$ that describes the initial quantum state $\mid \phi(\mathrm{x})$ i. The encoding techniques are described below:

- Basis Encoding: - In this type of encoding inputs say ' $\mathrm{x}$ ' is encoded to binary strings into the basis state of Qubits[21,22]. For Example $\mathrm{x}=01001$ is represented by the 5-qubit basis state |01001i. The computational basis state corresponds to a standard basis vector |ii (with $\mathrm{i}$ being the integer representation of the bit string) in a 2 -dimensional Hilbert space $F$, and thus, the effect of the featureembedding circuit is given by $\mathrm{U} \varphi: \mathrm{x} \in\{0,1\} \mathrm{n} \rightarrow \mid$ ii. This feature map maps each data input to a state from an orthonormal basis.

- Amplitude Encoding:-In this type of encoding we normalize the inputs ' $\mathrm{x}$ ' such that $\mathrm{x}=(\mathrm{x} 0, \ldots, \mathrm{xN}-1) \mathrm{T}$ $\in \mathrm{RN}$ of dimension $\mathrm{N}=2 \mathrm{n}$ with the amplitudes $\mid \psi \mathrm{xi}$ $[8,13], \mathrm{U} \varphi: \mathrm{x} \in \mathrm{RN} \rightarrow \mid \psi \mathrm{xi}=\mathrm{N}-1 \mathrm{X} \mathrm{i}=0$ xi|ii. Please find that, |ii denotes the $\mathrm{i}$ 'th computational basis state. This represents the linear kernel, $\kappa(\mathrm{x}, \mathrm{x} 0)=$ $\mathrm{h} \psi \mathrm{x} \mid \psi \mathrm{x} 0 \mathrm{i}=\mathrm{xT} \mathbf{x} 0$.

- Product Encoding: - This type of encoding uses tensor product encoding where features which have been encoded of the ' $x$ ' input depicted by $(\mathrm{x} 1, . ., \mathrm{xN}) \mathrm{T} \in \mathrm{RN}$ is encoded into Qubit amplitudes.

\section{B. Model Circuit}

In modelling Quantum circuits the 'ket' vector present in the Hilbert vector space maps to the ket vector $\phi 0=\mathrm{U} \theta \phi(\mathrm{x})$ by a unitary operation $\mathrm{U} \theta$, parameterized by a set of variables $\theta$. Now to train our VQC model I will formulate rules for parameters that will be looked up during optimization phase and learned, this may have an effect on the shape of the cost function. A single qubit gate $\mathrm{G}$ is a $2 \times 2$ unitary, which can always be written as $\mathrm{G}(\alpha, \beta, \gamma, \varphi)=\operatorname{ei} \varphi(\mathrm{ei} \beta \cos \alpha$ ei $\gamma \sin \alpha-\mathrm{e}-\mathrm{i} \gamma$ $\sin \alpha \mathrm{e}-\mathrm{i} \beta \cos \alpha)$ and may be defined by 4 parameters such as $\{\alpha, \beta, \gamma, \varphi\}$ [30].The number of Quantum gates depend linearly on the number of Qubit Architecture. This means the algorithm running in the VQC will require a polynomial amount of inputs, for this reason we will have a circuit architecture with rigid gates and controls. A number of combinations of Quantum circuit gates were used to mimic multi-qubit-based quantum classifiers but this is theoretical and a large number of such combinations may exists in reality $[10,13$, and 20]. 


\section{Measurement}

Measurement involve us measuring the output Qubits from got from the previous stages of processing and give us the required prediction on classifying the Iris flower into its respective genus. The pre-processing used in the classical methods is to sum a informative bias ' $b$ ' to extrapolate a sequential output such as $\pi(\mathrm{x} ; \theta, \mathrm{b})=\mathrm{p}(\mathrm{q} 0=1, \mathrm{x}, \theta)+\mathrm{b}$ such that we may assign a rule $\mathrm{f}(\mathrm{x} ; \theta)=\{1$ if $\pi(\mathrm{x} ; \theta)\}>0.5$ else 0 . This is to predict all given probabilities of 'pa' corresponding to outputs 'S3' for the state $\psi(\mathrm{x})$ i. pa $=\operatorname{Tr}(\pi \mathrm{a})^{\sim} \psi(\mathrm{x})^{\sim} \psi(\mathrm{x}) \mid$, this will mean the probabilities of $\mathrm{S} 3=\mathrm{a} ; \mathrm{S} 3=\mathrm{PN}$ a $=0$ a $\pi$ a. I will be able to formulate a rule for our VQC so as to get $\mathrm{pb}=$ $\max \{\mathrm{pa}\} \rightarrow \mathrm{f} *(\mathrm{x})=$ ' $\mathrm{lb}$ ', this will inference the label ' $\mathrm{LB}$ ' will have $\mathrm{S} 3=\mathrm{b}$ as the most probable result from measuring our VQC. To reduce the noise spoken about earlier I need to Train and the VQC and optimize it using new parameter rules after every iteration or also known as Vector Matrix Multiplication or just VMM such that each weight in the matrix is adjustable based on the parameter changes and can be modified during training[8]. This may be also understood as a VMM operation because of the latter process.

\section{III.CONCLUSION}

Our paper presents a review of existing classical-quantum hybrid algorithms, mainly the Variational Quantum Classifier (VQC) and the steps required to implement it as part of Quantum Circuit Learning and serves as a beacon for further research in the field of Quantum Computing and Quantum Machine Learning. In our demonstration of VQC for Iris Dataset we have shown that state preparation will encode the classical data set into quantum amplitudes so as to make it understandable by the quantum hardware by one of the methods, next we demonstrate how the processes will be performed by VQC by Unitary operations which involves Quantum theory knowledge with the help of non-mutable parameters for the algorithm. As a result we will measure the Qubits received from performing previous processes to actually get the prediction for classification of the Iris Flower into it correct biological genus. This signifies a hybrid algorithm which harnesses Quantum hardware and classical machine learning algorithms. Hence this states that my team and I were in fact able to deploy classical Machine Learning which can be interpreted by Quantum hardware.

\section{REFERENCES}

[1] Kak S. On quantum neural computing. Inf. Sci. (NY) 83, 143160,(1995)J.

[2] Servedio RA, Gortler SJ. Equivalences and separations between quantum and classical learnability. SIAM J. Comput. 33, 10671092,(2004)

[3] Atici A, Servedio RA. Improved bounds on quantum learning algorithms. Quantum Inf. Process. 4, 355-386, (2011)

[4] Childs AM. Quantum algorithms: equation solving by simulation. Nat. Phys. 5, 861-861, (2011)

[5] Hen I, Young A. Exponential complexity of the quantum adiabatic algorithm for certain satisfiability problems. Phys. Rev. E 84, 061152 (2011)

[6] Schwarz M, Temme K, Verstraete F. Preparing projected entangled pair states on a quantum computer. Phys. Rev. Lett. 108,110502,(2012)

[7] Schwarz M, Temme K, Verstraete F. Preparing projected entangled pair states on a quantum computer. Phys. Rev. Lett. 108, 110502 (2012)

[8] Jacob Biamonte, Peter Wittek, Nicola Pancotti, Patrick Rebentrost, Nathan Wiebe, and Seth Lloyd. Quantum machine learning. Nature, 549(7671):195, (2017)

[9] Patrick Rebentrost, Masoud Mohseni, and Seth Lloyd. Quantum support vector machine for big data classification. Physcial Review Letters, 113:130503 (2014)

[10] Iordanis Kerenedis and Anupam Prakash. Quantum recommendation systems. arXiv preprint arXiv:1603.08675, (2016)

[11] Nathan Wiebe, Daniel Braun, and Seth Lloyd. Quantum algorithm for data fitting. Physical Review Letters, 109(5):050505, (2012)

[12] Maria Schuld, Mark Fingerhuth, and Francesco Petruccione. Implementing a distance-based classifier with a quantum interference circuit. EPL (Europhysics Letters) (2017)

[13] J. Preskill, Quantum 2, 79, (2018)

[14] J. R. McClean, J. Romero, R. Babbush, and A. AspuruGuzik, New J. Phys. 18, 023023 (2016)

[15] Jeremy Adcock, Euan Allen, Matthew Day, Stefan Frick, Janna Hinchliff, Mack Johnson, Sam Morley-Short, Sam Pallister, Alasdair Price, Stasja Stanisic. Advances in Quantum Machine Learning. arXiv: 1512.02900 (2014)

[16] New Journal of Physics (2016) Jarrod R. McClean, Jonathan Romero, Ryan Babbush, Alán Aspuru-Guzik. The theory of variational hybrid quantum-classical algorithms. arXiv:1509.04279 (2015)

[17] Havl'1'cek V, C'orcoles A D, Temme K, Harrow A W, Kandala A, Chow J M and Gambetta J M, Nature 567, 209 (2019)

[18] McClean J R, Romero J, Babbush R and Aspuru-Guzik A New Journal of Physics 18023023 (2016)

[19] ]. Arunachalam S, de Wolf R. 2017. Optimal quantum sample complexity of learning algorithms. In Proc. 32nd Computational Complexity Conference, CCC 2017, Riga, Latvia, 6-9 (2017)

[20] Schuld M, Bocharov A, Svore K and Wiebe N, arXiv preprint arXiv: 1804.00633 (2018)

[21] E. Farhi and H. Neven, arXiv preprint arXiv:1802.06002 (2018)

[22] S. Wang, Journal of Mathematics Research 7, 175 (2015) 